\title{
A maximally-graded invertible cubic threefold that does not admit a full exceptional collection of line bundles
}

\author{
David Favero $^{1}$, Daniel Kaplan ${ }^{2}$ and Tyler L. Kelly ${ }^{3}$ \\ ${ }^{1}$ University of Alberta, Department of Mathematical and Statistical Sciences, Central Academic Building 632, Edmonton, AB, \\ Canada T6G 2C7 \\ Korea Institute for Advanced Study, 85 Hoegiro, Dongdaemun-gu, Seoul, Republic of Korea 02455; E-mail: favero@ualberta.ca. \\ ${ }^{2}$ University of Birmingham, School of Mathematics, Birmingham B15 2TT, United Kingdom; E-mail: d.kaplan@bham.ac.uk. \\ ${ }^{3}$ University of Birmingham, School of Mathematics, Birmingham B15 2TT, United Kingdom; E-mail: t.kelly.1@bham.ac.uk.
}

Received: 30 April 2020; Revised: 5 May 2020; Accepted: 25 May 2020

Keywords and phrases: exceptional collections; derived categories; tilting object

2020 Mathematics Subject Classification: Primary - 14F08

\begin{abstract}
We show that there exists a cubic threefold defined by an invertible polynomial that, when quotiented by the maximal diagonal symmetry group, has a derived category that does not have a full exceptional collection consisting of line bundles. This provides a counterexample to a conjecture of Lekili and Ueda.
\end{abstract}

\section{Contents}

1 Introduction $\quad 1$

1.1 Relation to current literature and mirror symmetry . . . . . . . . . . . . 2

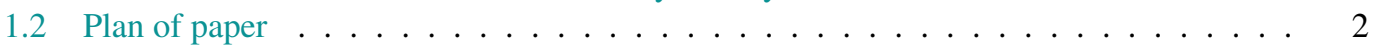

2 Line bundles on $X_{w} \quad 3$

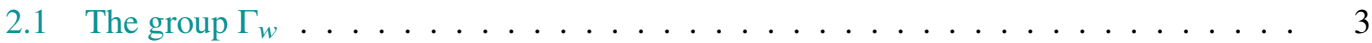

2.2 The Picard group of $X_{w} \ldots \ldots \ldots \ldots \ldots \ldots \ldots$

3 Dimension of the Hochschild homology of $\mathrm{D}^{\mathrm{b}}\left(\operatorname{coh} X_{w}\right) \quad 4$

4 Computations of Ext between line bundles on $X_{w} \quad 5$

\section{Introduction}

Let $\mathbb{C}$ be the complex numbers. We say a polynomial $w \in \mathbb{C}\left[x_{1}, \ldots, x_{n}\right]$ is invertible if it is of the form

$$
w=\sum_{i=1}^{n} \prod_{j=1}^{n} x_{j}^{a_{i j}}
$$

where $A=\left(a_{i j}\right)_{i, j=1}^{n}$ is a non-negative integer-valued matrix satisfying the following conditions:

\footnotetext{
(c) The Author(s), 2020. Published by Cambridge University Press. This is an Open Access article, distributed under the terms of the Creative Commons Attribution licence (http://creativecommons.org/licenses/by/4.0/), which permits unrestricted re-use, distribution, and reproduction in any medium, provided the original work is properly cited.
} 
A. The matrix $A$ is invertible over $\mathbb{Q}$;

B. The polynomial $w$ is quasihomogeneous: that is, there exist positive integers $q_{j}$ such that $d:=$ $\sum_{j=1}^{n} q_{j} a_{i j}$ is constant for all $i$; and

C. The polynomial $w$ is quasi-smooth: that is, the map $w: \mathbb{C}^{n} \rightarrow \mathbb{C}$ has a unique critical point at the origin.

Let $\mathbb{G}_{m}$ be the multiplicative torus. Consider the following group:

$$
\Gamma_{w}:=\left\{\left(t_{1}, \ldots, t_{n+1}\right) \in \mathbb{G}_{m}^{n+1} \mid w\left(t_{1} x_{1}, \ldots, t_{n} x_{n}\right)=t_{n+1} w\left(x_{1}, \ldots, x_{n}\right)\right\}
$$

This group $\Gamma_{w}$ acts on $\mathbb{A}^{n}$ by projecting onto its first $n$ coordinates and then acting diagonally. Lekili and Ueda made the following conjecture concerning the bounded derived category associated to the polynomial $w$ and the group $\Gamma_{w}$.

Conjecture 1.1 (Conjecture 1.3 of [20]). For any invertible polynomial $w$, the bounded derived category $\mathrm{D}^{\mathrm{b}}\left(\operatorname{coh} X_{w}\right)$ of coherent sheaves on the stack

$$
X_{w}:=\left[\left(\operatorname{Spec}\left(\mathbb{C}\left[x_{1}, \ldots, x_{n}\right] /(w)\right) \backslash 0 / \Gamma_{w}\right]\right.
$$

has a tilting object, which is a direct sum of line bundles.

In this paper, we show that

$$
w=x_{1}^{2} x_{2}+x_{2}^{2} x_{3}+x_{3}^{2} x_{4}+x_{4}^{2} x_{5}+x_{5}^{2} x_{1}
$$

provides a counterexample to this conjecture. In fact, the maximal length of any exceptional collection of line bundles on $\mathrm{D}^{\mathrm{b}}\left(\operatorname{coh} X_{w}\right)$ is 24 . On the other hand, we calculate that 54 line bundles would be required in any full exceptional collection, let alone a tilting object.

\subsection{Relation to current literature and mirror symmetry}

The result above is analogous to the case of toric varieties. It was asked by King if the derived category of a smooth projective toric variety admits a tilting object that is a direct sum of line bundles. This later became known as King's conjecture. The first counterexamples to King's conjecture were provided by Hille-Perling [10] and then later by Efimov [2] in the Fano case. Nevertheless, in [15], Kawamata proved that the derived category of any smooth projective toric Deligne-Mumford stack has a full exceptional collection. It just need not consist of line bundles (or sheaves, for that matter, see [16, Remark 7]).

The Landau-Ginzburg B-model analogue to $\mathrm{D}^{\mathrm{b}}\left(\operatorname{coh} X_{w}\right)$ given by the singularity category of $\left(\mathbb{C}^{n}, \Gamma_{w}, w\right)$ is well-studied in the context of homological mirror symmetry. At present, it is known to have a full exceptional collection [3]. It is also known to have a full strong exceptional collection in certain cases: for example, when $n \leq 3$ [18] or when $w$ can be written as the Thom-Sebastiani sum of Fermat and chain polynomials [12]. This has been desirable in order to establish homological mirror symmetry for mirror pairs of (gauged) Landau-Ginzburg models [4, 5, 8, 13, 14, 20, 21].

\subsection{Plan of paper}

In Section 2, we show that the Picard group of the stack $X_{w}$ is isomorphic to $\mathbb{Z} \times \mathbb{Z} / 11 \mathbb{Z}$. In Section 3, we calculate that the Chen-Ruan cohomology of $X_{w}$ is 54-dimensional. This implies that the cardinality of any full exceptional collection for $\mathrm{D}^{\mathrm{b}}\left(\operatorname{coh} X_{w}\right)$ must be 54 (Corollary 3.2). On the other hand, in Section 4, we find a sharp upper bound of 24 on the cardinality of an exceptional collection for $\mathrm{D}^{\mathrm{b}}\left(\operatorname{coh} X_{w}\right)$ consisting of line bundles. 


\section{Line bundles on $X_{w}$}

To address Conjecture 1.1, we first require an explicit description of the Picard group of $X_{w}$.

\subsection{The group $\Gamma_{w}$}

First, we define the group of diagonal automorphisms of the invertible polynomial $w$ to be

$$
G_{w}:=\left\{\left(t_{1}, \ldots, t_{n}\right) \in \mathbb{G}_{m}^{n} \mid w\left(t_{1} x_{1}, \ldots, t_{n} x_{n}\right)=w\left(x_{1}, \ldots, x_{n}\right)\right\} .
$$

This sits in an exact sequence

$$
0 \longrightarrow G_{w} \longrightarrow \Gamma_{w} \stackrel{\chi_{n+1}}{\longrightarrow} \mathbb{G}_{m} \rightarrow 0
$$

where $\chi_{n+1}$ is the projection onto the $(n+1)^{\text {th }}$ term of $\Gamma_{w}$. Indeed, we know that $\chi_{n+1}$ is surjective, as, given $\lambda \in \mathbb{G}_{m}$, we have that $\left(\lambda^{q_{1} / d}, \ldots, \lambda^{q_{n} / d}, \lambda\right) \in \Gamma_{w}$.

By Lemma 1.6(B) of [17] for a loop polynomial

$$
w=x_{1}^{a_{1}} x_{2}+x_{2}^{a_{2}} x_{3}+\ldots+x_{n-1}^{a_{n-1}} x_{n}+x_{n}^{a_{n}} x_{1},
$$

we have $G_{w} \cong \mathbb{Z} /\left(a_{1} \cdots a_{n}+(-1)^{n+1}\right) \mathbb{Z}$ with generator $\left(e^{2 \pi i \varphi_{1}}, \ldots, e^{2 \pi i \varphi_{n}}\right)$, where

$$
\varphi_{j}:=\frac{(-1)^{n+1-j} a_{1} \cdots a_{j-1}}{a_{1} \cdots a_{n}+(-1)^{n+1}} .
$$

Recall that $w$ is quasi-homogeneous: that is, we can choose $q_{i}$ such that $d:=\sum_{j=1}^{n} q_{j} a_{i j}$ is constant for all $i$ and such that $\operatorname{gcd}\left(q_{1}, \ldots, q_{n}\right)=1$. This yields a subgroup $J_{w} \cong \mathbb{G}_{m}$ defined by

$$
f: J_{w} \rightarrow \Gamma_{w} ; \quad f(\lambda)=\left(\lambda^{q_{1}}, \ldots, \lambda^{q_{n}}, \lambda^{d}\right)
$$

known as the exponential grading operator in the literature.

Furthermore, the inclusion $f$ gives rise to a split short exact sequence

$$
0 \longrightarrow J_{w} \longrightarrow \Gamma_{w} \longrightarrow \overline{G_{w}} \longrightarrow 0
$$

where $\overline{G_{w}}:=G_{w} /\left(J_{w} \cap G_{w}\right)$ is the quotient group. Since $\operatorname{gcd}\left(q_{1}, \ldots, q_{n}\right)=1$, there exist $b_{i}$ with $\sum_{i=1}^{n} b_{i} q_{i}=1$, which gives rise to the splitting of the exact sequence given by

$$
g: \Gamma_{w} \rightarrow J_{w} ; \quad g\left(\lambda_{1}, \ldots, \lambda_{n}, \lambda_{n+1}\right)=\prod_{i=1}^{n} \lambda_{i}^{b_{i}}
$$

Hence $\Gamma_{w} \cong J_{w} \times \overline{G_{w}}$.

The isomorphism $\Gamma_{w} \cong J_{w} \times \overline{G_{w}}$ gives rise to an intermediate quotient stack associated to $J_{w}$,

$$
Z_{w}=\left[\left(\operatorname{Spec}\left(\mathbb{C}\left[x_{1}, \ldots, x_{n}\right] /(w)\right) \backslash 0\right) / J_{w}\right]
$$

which is a hypersurface in the weighted projective stack

$$
\left[\left(\operatorname{Spec}\left(\mathbb{C}\left[x_{1}, \ldots, x_{n}\right]\right) \backslash 0\right) / J_{w}\right]=\mathbb{P}\left(q_{1}: \cdots: q_{n}\right) .
$$

This allows us to identify $X_{w}$ with the quotient $\left[Z_{w} / \overline{G_{w}}\right]$. 
Example 2.1. Let $w=x_{1}^{2} x_{2}+x_{2}^{2} x_{3}+x_{3}^{2} x_{4}+x_{4}^{2} x_{5}+x_{5}^{2} x_{1}$, as in (2). Then $G_{w}=\mathbb{Z} / 33 \mathbb{Z}$ with generator

$$
g=\left(\zeta, \zeta^{-2}, \zeta^{4}, \zeta^{-8}, \zeta^{16}\right)
$$

where $\zeta$ is a primitive 33rd root of unity. Here, the intersection $J_{w} \cap G_{w}$ is generated by $g^{11}=\left(\zeta^{11}, \zeta^{11}, \zeta^{11}, \zeta^{11}, \zeta^{11}\right)$. Hence $\overline{G_{w}}$ can be identified with the symmetry group generated by $\left(\xi, \xi^{9}, \xi^{4}, \xi^{3}, \xi^{5}\right)$, where $\xi$ is a primitive 11th root of unity.

\subsection{The Picard group of $X_{w}$}

The Grothendieck-Lefschetz theorem allows us to calculate the Picard group of $X_{w}$ as follows.

Proposition 2.2. Let $w$ be an invertible polynomial with $n \geq 5$ and $q_{1}=\ldots=q_{n}=1$. The Picard group of $X_{w}$ is isomorphic to $\mathbb{Z} \times \overline{\overline{G_{w}}}$, where $\overline{\overline{G_{w}}}$ is the group of characters of $\overline{G_{w}}$.

Proof. Since $X_{w}=\left[Z_{w} / \overline{G_{w}}\right]$ is a global quotient stack, $\operatorname{Pic}\left(X_{w}\right)$ is nothing more than the $\overline{G_{w}}$ equivariant Picard group of $Z_{w}$. Note that there is a (surjective) pullback map

$$
\operatorname{Pic}\left(X_{w}\right) \stackrel{f}{\rightarrow} \operatorname{Pic}\left(Z_{w}\right)
$$

that just forgets the equivariant structure. By the Grothendieck-Lefschetz theorem (see, for example, [9, Corollary 3.2]), $\operatorname{Pic}\left(Z_{w}\right) \cong \mathbb{Z}$ : that is, any line bundle is of the form $\mathcal{O}(n)$. As $\mathcal{O}(n)$ admits an equivariant structure, the forgetful map $f$ is surjective.

Furthermore, as any two equivariant structures differ by a character of $\overline{G_{w}}$, we get a short exact sequence

$$
0 \longrightarrow \widehat{\overline{G_{w}}} \longrightarrow \operatorname{Pic}\left(X_{w}\right) \stackrel{f}{\longrightarrow} \mathbb{Z} \longrightarrow 0
$$

Since $\mathbb{Z}$ is a projective $\mathbb{Z}$-module, this splits to give the desired isomorphism.

Example 2.3. Let $w=x_{1}^{2} x_{2}+x_{2}^{2} x_{3}+x_{3}^{2} x_{4}+x_{4}^{2} x_{5}+x_{5}^{2} x_{1}$ so that $\overline{G_{w}}=\mathbb{Z} / 11 \mathbb{Z}$. Then by Proposition 2.2, we have $\operatorname{Pic}\left(X_{w}\right) \cong \mathbb{Z} \times(\mathbb{Z} / 11 \mathbb{Z})$.

\section{Dimension of the Hochschild homology of $\mathrm{D}^{\mathrm{b}}\left(\operatorname{coh} X_{w}\right)$}

In this section, we compute the dimension of the Chen-Ruan cohomology of $X_{w}$ to be 54. This implies that any full exceptional collection for $\mathrm{D}^{\mathrm{b}}\left(\operatorname{coh} X_{w}\right)$ must have 54 objects.

Proposition 3.1. Let $w=x_{1}^{2} x_{2}+x_{2}^{2} x_{3}+x_{3}^{2} x_{4}+x_{4}^{2} x_{5}+x_{5}^{2} x_{1}$. Then $\operatorname{dim}\left(H_{C R}^{*}\left(X_{w} ; \mathbb{C}\right)\right)=54$.

Proof. As vector spaces, the (ungraded) Chen-Ruan cohomology of $X_{w}$ is the direct sum of ordinary cohomology groups of twisted sectors

$$
H_{C R}^{*}\left(X_{w} ; \mathbb{C}\right)=\bigoplus_{\gamma \in \Gamma_{w}} H^{*}\left(\{w=0\}_{\gamma} / \Gamma_{w} ; \mathbb{C}\right)
$$

where $\{w=0\}_{\gamma}:=\left\{x \in\{w=0\}_{\mathbb{C}^{5} \backslash\{0\}} \mid \gamma \cdot x=x\right\}$ [1, Section 3].

First, note that if $\gamma=\left(\lambda_{1}, \ldots, \lambda_{5}\right)$ so that $\lambda_{i} \neq 1$ for all $i$, then $\gamma \cdot x \neq x$ for all $x \in \mathbb{C}^{5} \backslash\{0\}$. This implies that the twisted sector corresponding to $\gamma$ contributes the cohomology of the empty set: that is, nothing.

First, we address the twisted sector associated to the identity element $\gamma=e$. Note that $H^{*}(\{w=$ $\left.0\} / \Gamma_{w} ; \mathbb{C}\right)=H^{*}\left(Z_{w} ; \mathbb{C}\right)^{\bar{G}_{w}}$, so we must see how $\overline{G_{w}}$ acts on the cohomology of $Z_{w}$. Recall that the 
Hodge diamond of the cubic $Z_{w}$ is of the form

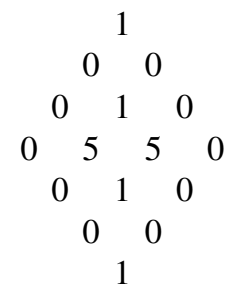

This is computed using the Griffiths' residue map [6], which also allows us to describe the action of $\overline{G_{w}}$. Namely, any element $H^{2,1}\left(Z_{w}\right)$ can be written as the residue of a 4-form

$$
\varphi=\frac{Q}{w} \Omega_{0}, \quad \Omega_{0}=\sum_{i=1}^{5}(-1)^{i} x_{i} \mathrm{~d} x_{1} \wedge \ldots \wedge \widehat{\mathrm{d} x_{i}} \wedge \ldots \wedge \mathrm{d} x_{5}
$$

where $Q$ is a degree 1 polynomial in $\mathbb{C}\left[x_{1}, \ldots, x_{5}\right]$. By looking at the action by the generator $\rho$ of $\overline{G_{w}}$, we can see that $w$ and $\Omega_{0}$ are invariant under its action; however, no degree 1 polynomial is invariant, so the $\overline{G_{w}}$-invariant subspace of $H^{2,1}\left(Z_{w} ; \mathbb{C}\right)$ is zero. Similarly, the $\overline{G_{w}}$-invariant subspace of $H^{1,2}\left(Z_{w} ; C\right)$ is zero. The hyperplane classes, on the other hand, are all invariant cycles, so

$$
\operatorname{dim} H^{*}\left(\{w=0\} / \Gamma_{w} ; \mathbb{C}\right)=4 .
$$

Lastly, there are 50 non-identity elements

$$
S:=\left\{\left(\rho \tau^{-1}\right)^{a},\left(\rho \tau^{-9}\right)^{a},\left(\rho \tau^{-4}\right)^{a},\left(\rho \tau^{-3}\right)^{a},\left(\rho \tau^{-5}\right)^{a} \mid 1 \leq a \leq 10\right\} \subseteq \Gamma_{w}
$$

with a fixed point where $\rho:=\left(\xi, \xi^{9}, \xi^{4}, \xi^{3}, \xi^{5}\right)$ is the generator of $\overline{G_{w}}$ and $\tau=(\xi, \xi, \xi, \xi, \xi)$. In fact, each has a single fixed point and hence contributes one dimension to the Chen-Ruan cohomology.

We conclude that $\operatorname{dim}\left(H_{C R}^{*}\left(X_{w} ; \mathbb{C}\right)\right)=4+|S|=4+50=54$.

This proposition implies the following corollary.

Corollary 3.2. For $w$ as defined in (2), we have that $\operatorname{dim}\left(H H_{*}\left(\mathrm{D}^{\mathrm{b}}\left(\operatorname{coh} X_{w}\right)\right)\right)=54$. In particular, any full exceptional collection for $\mathrm{D}^{\mathrm{b}}\left(\operatorname{coh} X_{w}\right)$ has precisely 54 objects.

Proof. By an unpublished result of Toën (reproven in [7, Proposition 3.16]),

$$
\operatorname{dim}\left(\mathrm{HH}_{*}\left(\mathrm{D}^{\mathrm{b}}\left(\operatorname{coh} X_{w}\right)\right)\right)=\operatorname{dim}\left(H_{C R}^{*}\left(X_{w} ; \mathbb{C}\right)\right)=54 .
$$

The fact that any full exceptional collection must have 54 objects follows from the additivity of Hochschild homology under semi-orthogonal decomposition.

Remark 3.3. In [3, Theorem 1.1], the authors prove that there is a strong exceptional collection for the singularity category $\mathrm{D}\left[\mathbb{A}^{5}, \Gamma_{w}, w\right]$. It is of length 32 , the Milnor number of its mirror LG-model. By the equivariant version of Orlov's theorem (proven by Hirano [11, Theorem 1.3]), it follows that $\mathrm{D}^{\mathrm{b}}\left(\operatorname{coh} X_{w}\right)$ has a full exceptional collection of length $32+2(11)=54$. From this, it also follows that any full exceptional collection must have 54 objects.

\section{Computations of Ext between line bundles on $X_{w}$}

By Corollary 3.2, any full exceptional collection for $\mathrm{D}^{\mathrm{b}}\left(\operatorname{coh} X_{w}\right)$ has 54 objects. However, in this section, we show that an exceptional collection consisting of line bundles on $X_{w}$ has at most 24 objects (and remark that this bound is achieved). 
Table 1. The $(a, b)$ th entry is an $(a, b)$-bigraded monomial in $\mathbb{C}\left[x_{1}, x_{2}, x_{3}, x_{4}, x_{5}\right] /(w)$.

\begin{tabular}{|c|c|c|c|c|c|c|c|c|c|c|c|}
\hline \multirow[b]{2}{*}{$\mathbb{Z}$-grading } & \multicolumn{11}{|c|}{$\mathbb{Z} / 11 \mathbb{Z}$-grading } \\
\hline & 0 & 1 & 2 & 3 & 4 & 5 & 6 & 7 & 8 & 9 & 10 \\
\hline 0 & 1 & & & & & & & & & & \\
\hline 1 & & $x_{1}$ & & $x_{4}$ & $x_{3}$ & $x_{5}$ & & & & $x_{2}$ & \\
\hline 2 & & $x_{2} x_{4}$ & $x_{1}^{2}$ & $x_{5} x_{2}$ & $x_{1} x_{4}$ & $x_{1} x_{3}$ & $x_{1} x_{5}$ & $x_{3} x_{4}$ & $x_{3}^{2}$ & $x_{3} x_{5}$ & $x_{1} x_{2}$ \\
\hline 3 & $x_{1}^{2} x_{2}$ & $x_{3}^{3}$ & $x_{1} x_{2} x_{4}$ & $x_{1}^{3}$ & $x_{1} x_{2} x_{5}$ & $x_{1}^{2} x_{4}$ & $x_{1}^{2} x_{3}$ & $x_{1}^{2} x_{5}$ & $x_{1} x_{3} x_{4}$ & $x_{1} x_{3}^{2}$ & $x_{1} x_{3} x_{5}$ \\
\hline
\end{tabular}

Lemma 4.1. For $a \geq 0, \operatorname{Hom}(\mathcal{O}, \mathcal{O}(a, b)) \neq 0$ unless $a=0$ and $b \neq 0$ or

$$
(a, b) \in \mathbb{X}:=\{(1,0),(1,2),(1,6),(1,7),(1,8),(1,10),(2,0)\} .
$$

Proof. Observe that $\operatorname{Hom}(\mathcal{O}, \mathcal{O}(a, b))$ is the space of bidegree $(a, b) \in \mathbb{Z} \times \mathbb{Z} / 11 \mathbb{Z}$ polynomials in $\mathbb{C}\left[x_{1}, x_{2}, x_{3}, x_{4}, x_{5}\right] /(w)$. By Example $2.1, \bar{G}_{w}=\left\langle\left(\xi, \xi^{9}, \xi^{4}, \xi^{3}, \xi^{5}\right)\right\rangle \cong \mathbb{Z} / 11 \mathbb{Z}$, where $\xi$ is a primitive 11 th root of unity. Hence,

$$
\operatorname{deg}\left(x_{1}\right)=(1,1), \operatorname{deg}\left(x_{2}\right)=(1,9), \operatorname{deg}\left(x_{3}\right)=(1,4), \operatorname{deg}\left(x_{4}\right)=(1,3), \operatorname{deg}\left(x_{5}\right)=(1,5) .
$$

So Table 1 exhibits an element in $\operatorname{Hom}(\mathcal{O}, \mathcal{O}(a, b))$ for $1 \leq a \leq 3$, unless $(a, b) \in \mathbb{X}$. We conclude that $\operatorname{Hom}(\mathcal{O}, \mathcal{O}(a, b))$ is non-zero for $a \geq 3$ by multiplying any monomial in $\operatorname{Hom}(\mathcal{O}, \mathcal{O}(3, b-a+3))$ by $x_{1}^{a-3}$.

Lemma 4.2. For $a \geq 2$, we have that $\operatorname{Ext}^{3}(\mathcal{O}(a, b), \mathcal{O}) \neq 0$ unless $a=2$ and $b \neq 0$ or

$$
(a, b) \in \mathbb{X}^{\prime}:=\{(3,0),(3,2),(3,6),(3,7),(3,8),(3,10),(4,0)\} .
$$

Proof. By adjunction, the canonical bundle is $\mathcal{O}(-2,0)$. Therefore by Serre duality,

$$
\begin{aligned}
& \operatorname{Ext}^{i}(\mathcal{O}(a, b), \mathcal{O}) \stackrel{\text { Serre }}{\cong} \operatorname{Ext}^{3-i}\left(\mathcal{O}, \mathcal{O}(a, b) \otimes_{\mathcal{O}} \mathcal{O}(-2,0)\right)^{*} \\
&\left.\cong \operatorname{Ext}^{3-i}(\mathcal{O}, \mathcal{O}(a-2, b))\right)^{*}
\end{aligned}
$$

The result follows from Lemma 4.1.

Proposition 4.3. An exceptional collection of line bundles in $\mathrm{D}^{\mathrm{b}}\left(\operatorname{coh} X_{w}\right)$ has at most 24 objects and hence cannot be full (by Corollary 3.2).

Proof. By Example 2.3, any line bundle on $X_{w}$ is of the form $\mathcal{O}(a, b)$ for $(a, b) \in \mathbb{Z} \times \mathbb{Z} / 11 \mathbb{Z}$. Let $\mathcal{E}$ denote an exceptional collection of line bundles, and take the minimal $a$ such that $\mathcal{O}(a, b) \in \mathcal{E}$ for some $b \in \mathbb{Z} / 11 \mathbb{Z}$. Since $\mathcal{E} \otimes \mathcal{O}(-a,-b)$ is an exceptional collection, we can assume $(a, b)=(0,0)$.

Notice that $\mathcal{E}$ cannot have an object of the form $\mathcal{O}(a, b)$ for $a \geq 5$, as, by Lemma 4.1, $\mathcal{O}(a, b)$ receives a non-zero map from $\mathcal{O}$ and, by Lemma 4.2, there is a non-trivial 3-extension of $\mathcal{O}$ by $\mathcal{O}(a, b)$.

By Table 1 , observe that if $b \neq b^{\prime}$, then for any $a$, one has non-zero elements

$$
f_{1} \in \operatorname{Hom}\left(\mathcal{O}(a, b), \mathcal{O}\left(a+2, b^{\prime}\right)\right) \quad \text { and } \quad f_{2} \in \operatorname{Hom}\left(\mathcal{O}\left(a, b^{\prime}\right), \mathcal{O}(a+2, b)\right)
$$

Therefore, denoting by $\mathbb{S}(a, b)$ the Serre functor applied to the identity map on $\mathcal{O}(a, b)$, one has a loop:

$$
\mathcal{O}(a, b) \stackrel{f_{1}}{\longrightarrow} \mathcal{O}\left(a+2, b^{\prime}\right) \stackrel{\mathbb{S}\left(a+2, b^{\prime}\right)}{\longrightarrow} \mathcal{O}\left(a, b^{\prime}\right) \stackrel{f_{2}}{\longrightarrow} \mathcal{O}(a+2, b) \stackrel{\mathbb{S}(a+2, b)}{\longrightarrow} \mathcal{O}(a, b) .
$$


We conclude that $\mathcal{E}$ cannot have a quadruple of objects

$$
\left\{\mathcal{O}(a, b), \mathcal{O}\left(a, b^{\prime}\right), \mathcal{O}(a+2, b), \mathcal{O}\left(a+2, b^{\prime}\right)\right\} .
$$

For example, taking $a=0$ (respectively, $a=1$ ), $\mathcal{E}$ cannot have multiple objects with $a=0$ and $a=2$ (respectively, $a=1$ and $a=3$ ). This forces there to be at most 12 line bundles in $\mathcal{E}$ with $a=0,2$ and $a=1,3$, respectively.

Now, again by Lemma 4.2, $\mathcal{E}$ cannot have an object of the form $\mathcal{O}(a, b)$ for $a \geq 4$ except $(a, b)=(4,0)$. Hence, we can have at most 1 more object. But if $\mathcal{O}(4,0) \in \mathcal{E}$, Lemma 4.2 also forces $\mathcal{O}(0, b) \notin \mathcal{E}$ for $b \neq 0$. Hence, if we already have 12 line bundles in $\mathcal{E}$ with $a=0,2$ then $\mathcal{O}(2, b) \in \mathcal{E}$ for all $b$. This gives a contradiction, as $\mathcal{O}, \mathcal{O}(2,0), \mathcal{O}(4,0)$ also form a loop

$$
\mathcal{O} \stackrel{x_{1}^{2} x_{3} x_{5}}{\longrightarrow} \mathcal{O}(4,0) \stackrel{\mathbb{S}(4,0)}{\longrightarrow} \mathcal{O}(2,0) \stackrel{\mathbb{S}(2,0)}{\longrightarrow} \mathcal{O}
$$

and therefore cannot be in the same exceptional collection. We conclude that this 1 additional object cannot take us beyond 24 exceptional objects.

Remark 4.4. The upper bound of 24 exceptional objects is sharp. It is achieved by the exceptional collection drawn below. This exceptional collection is not strong, however; we only draw the degree- 0 maps for aesthetic simplicity. The required vanishing can be checked using Lemmas 4.1 and 4.2 and the fact that $\mathrm{Ext}^{1}, \mathrm{Ext}^{2}$ vanish for line bundles on a 3 -fold hypersurface in projective space (for example, using the long exact sequence for the divisor).

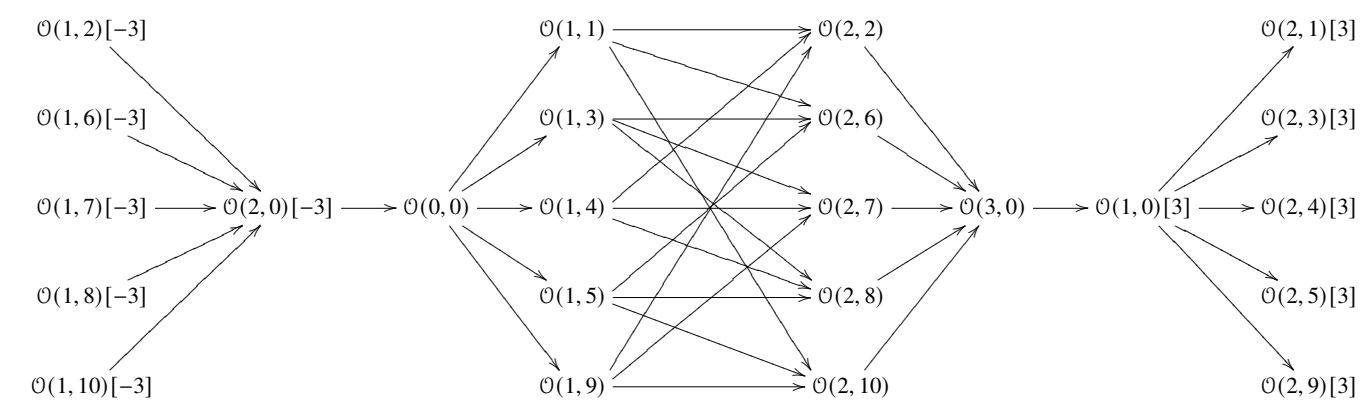

Acknowledgements. We cordially thank Yank Lekili, Atsushi Takahashi, Yuki Hirano, and Kazushi Ueda for their communications regarding the topic of this paper. We are especially grateful to Kazushi Ueda for spotting an issue with our initial attempt at finding a counterexample.

This project was initiated while the three authors visited the Fields Institute during the Thematic Program on the Homological Algebra of Mirror Symmetry. David Favero was supported by the Natural Sciences and Engineering Research Council of Canada through the Canada Research Chair and Discovery Grant programs. Daniel Kaplan was supported by the Engineering and Physical Sciences Research Council (EPSRC) under Grant EP/S03062X/1. Tyler Kelly was also supported by the EPSRC under Grant $\mathrm{EP} / \mathrm{N} 004922 / 2$ and EP/S03062X/1.

\section{References}

[1] A. Chiodo and Y. Ruan, 'LG/CY correspondence: the state space isomorphism', Adv. Math. 227(6): 2157-2188 (2011).

[2] A. I. Efimov, 'Maximal lengths of exceptional collections of line bundles', J. Lond. Math. Soc. 90(2): 350-372 (2014).

[3] D. Favero, D. Kaplan, and T. L. Kelly, 'Exceptional collections for mirrors of invertible polynomials', arxiv:2001:06500.

[4] M. Futaki and K. Ueda, 'Homological mirror symmetry for Brieskorn-Pham singularities', in Proceedings of the 56th Japan Geometry Symposium (Saga University, 2009), 98-107.

[5] M. Futaki and K. Ueda, 'Homological mirror symmetry for Brieskorn-Pham singularities', Selecta Math. (N.S.) 17(2): 435-452 (2011).

[6] P. Griffiths, 'On periods of certain rational integrals: I', Ann of Math. 90: 460-495 (1969).

[7] D. Halpern-Leistner and D. Pomerleano, 'Equivariant Hodge theory and noncommutative geometry', arxiv:1507.01924v2.

[8] M. Habermann and J. Smith, 'Homological Berglund-Hübsch mirror symmetry for curve singularities', arxiv:1903.01351. 
[9] R. Hartshorne, 'Ample subvarieties of algebraic varieties', Springer Lect. Notes Math. 156 (1970).

[10] L. Hille and M. Perling, 'A counterexample to King's conjecture', Compos. Math. 142(6): 1507-1521 (2006).

[11] Y. Hirano, 'Derived Knörrer periodicity and Orlov's theorem for Gauged Landau-Ginzburg models', Compos. Math. 153: 973-1007 (2017).

[12] Y. Hirano and G. Ouchi, 'Derived factorization categories of non-Thom-Sebastiani-type sum of potentials', arxiv:1809.09940.

[13] H. Kajiura, K. Saito, and A. Takahashi, 'Matrix factorizations and representations of quivers II. Type ADE case', Adv. Math. 211(1): 327-362 (2007).

[14] H. Kajiura, K. Saito, and A. Takahashi, 'Triangulated categories of matrix factorizations for regular systems of weights with $\epsilon=-1$ ', Adv. Math. 220(5): 1602-1654 (2009).

[15] Y. Kawamata, 'Derived categories of toric varieties', Michigan Math. J. 54(3): 517-535 (2006).

[16] Y. Kawamata, 'Derived categories of toric varieties II', Michigan Math. J. 62(2): 353-363 (2013).

[17] M. Krawitz, 'FJRW rings and Landau-Ginzburg mirror symmetry', arxiv:0906.0796.

[18] O. Kravets, 'Categories of singularities of invertible polynomials', arxiv:1911.09859.

[19] M. Kreuzer and H. Skarke, 'On the classification of quasihomogeneous functions', Comm. Math. Phys. 150(1): 137-147 (1992).

[20] Y. Lekili and K. Ueda, 'Homological mirror symmetry for Milnor fibers via moduli of $A_{\infty}$-structures', arxiv: $1806.04345 \mathrm{v} 2$.

[21] A. Takahashi, 'HMS for isolated hypersurface singularities', Workshop on Homological Mirror Symmetry and Related Topics, 19-24 January 2009, University of Miami, http://www-math.mit.edu/ auroux/frg/miami09-notes/. 